\title{
Polimorfismos de los genes calpaína y calpastatina en el ganado criollo colombiano Hartón del Valle
}

\author{
Polymorphisms of the Calpain and Calpastatin genes in the Colombian CReole \\ HaRTón del VALLe CATTLE
}

\author{
Claudia Lenis ${ }^{1}$, Liliana Ramos ${ }^{1}$, Melina Londoño ${ }^{1}$, Darwin Hernández ${ }^{2,3}$, \\ Luz Álvarez ${ }^{1}$
}

\section{ResUmen}

El objetivo del presente trabajo fue caracterizar el polimorfismo de nucleótido simple 316 y 4751 del gen CAPN y 2959 y CAST1 del gen CAST en la raza bovina criolla colombiana Hartón del Valle. Se genotiparon 126 individuos mediante PCR-RFLP para los cuatro loci. Todos los marcadores fueron polimórficos. El genotipo CC del locus CAPN316 y el heterocigoto en los otros tres loci tuvieron mayor frecuencia. Las frecuencias de los alelos asociados positivamente con calidad de la carne fueron de $\mathrm{C}=21.8 \pm 17.5 \% \mathrm{en}$ CAPN-316, $\mathrm{C}=48.4 \pm 10.1 \%$ en CAPN-4751, $\mathrm{T}=52.5 \pm 11.4 \%$ en CAST- 1 y A $=65.1 \pm$ $15.5 \%$ en CAST-2959. En todo los loci, la Ho $(0.599 \pm 0.248)$ fue más alta que la He $(0.410$ $\pm 0.081)$ con valores negativos de $\mathrm{F}_{\mathrm{IS}} \mathrm{y} \mathrm{F}_{\mathrm{IT}} \mathrm{y}$ con desviaciones de EHW solo en CAPN4751. El valor de $\mathrm{F}_{\mathrm{ST}}$ fue de $0.048(\mathrm{p}<0.05)$ y el AMOVA mostró que la variación en estos loci se debía en mayor parte a los individuos (97\%). Lo anterior puede ser indicativo de que esta raza presenta predisposición genética para la producción de carne con perfiles de calidad.

Palabras clave: calidad de carne; PCR-RFLP; polimorfismo de nucleótido simple; recursos zoogenéticos

\section{Abstract}

The objective of the present work was to characterize the simple nucleotide polymorphism 316 and 4751 of the CAPN and 2959 gene and CAST 1 of the CAST gene in the Colombian creole breed Hartón del Valle. A total of 126 individuals were genotyped

\footnotetext{
${ }^{1}$ Grupo de Investigación en Recursos Zoogenéticos, Facultad de Ciencias Agropecuarias, Universidad Nacional de Colombia, Sede Palmira, Colombia

${ }^{2}$ Grupo de Investigación en Reproducción y Mejoramiento Genético Animal, Departamento de Zootecnia, Facultad de Ciencias Agropecuarias, Universidad de Sucre - Campus Ciencias Agropecuarias, Sincelejo, Colombia

${ }^{3}$ E-mail: darwin.hernandez@unisucre.edu.co
} 
by PCR-RFLP for the four loci. All markers were polymorphic. The CC genotype of the CAPN-316 locus and the heterozygote in the other three loci were more frequent. The frequencies of the alleles positively associated with meat quality were $\mathrm{C}=21.8 \pm 17.5 \%$ in CAPN-316, C $=48.4 \pm 10.1 \%$ in CAPN-4751, $\mathrm{T}=52.5 \pm 11.4 \%$ in CAST- 1 and $\mathrm{A}=65.1 \pm$ $15.5 \%$ in CAST-2959. In all the loci the $\mathrm{Ho}(0.599 \pm 0.248)$ was higher than the $\mathrm{He}(0.410 \pm$ 0.081 ) with negative values of $\mathrm{F}_{\mathrm{IS}}$ and $\mathrm{F}_{\mathrm{IT}}$ and with deviations of EHW only in CAPN4751. The $\mathrm{F}_{\mathrm{ST}}$ value was $0.048(\mathrm{p}<0.05)$ and the AMOVA showed that the variation in these loci was mostly due to individuals (97\%). This may be indicative that this breed has a genetic predisposition for meat production of better quality.

Key words: meat quality; simple nucleotide polymorphism; PCR-RFLP; zoogenetic resources

\section{INTRODUCCIÓN}

El color, la grasa intramuscular, el área del ojo de lomo y la palatabilidad son los atributos que determinan la calidad de la carne. La palatabilidad, definida como la serie de sensaciones experimentadas por el consumidor, está relacionado con la terneza, la jugosidad y el sabor. Entre estas, sin duda, la terneza es la más privilegiada por el consumidor como criterio de calidad (Li et al., 2013). La terneza es un atributo complejo, en el cual intervienen factores como la edad, sexo, raza, stress del animal pre-faenado, condiciones de sacrificio, actividad proteolítica pos-mortem, contenido y densidad de fibra en el músculo, y cantidad, tipo y disposición del tejido conectivo (Chriki et al., 2013).

La degradación proteolítica pos-mortem ocurre durante la conversión de músculo a carne en el periodo de almacenamiento a temperaturas de refrigeración y es regulada principalmente por el sistema calpaína (CAPN) - calpastatina (CAST) (Ba et al., 2015); por tanto, polimorfismos de un solo nucleótido (SNP) en estos genes han sido asociados con terneza de la carne (White et al., 2005; Allais et al., 2011; Sava ${ }^{\circ}$ ç y Atasoy, 2016), aunque también con características reproductivas y de producción de leche (Hill et al., 2016).
El gen CAPN se encuentra en el cromosoma 29 bovino y posee 21 exones, con una longitud de $2255 \mathrm{pb}$. La proteína posee 716 aminoácidos y se han reportado 276 SNPs (ENSEMBL, 2017). Las calpaínas constituyen una amplia super familia de proteinasas intracelulares citosólicas dependientes de calcio, con diferentes isoformas, las cuales se encuentran ampliamente distribuidas en todos los tejidos y muestran una actividad regulada por el $\mathrm{pH}$. Las calpaínas están constituidas, sin excepción, por dos subunidades asociadas de tal manera que cada calpaína es un heterodímero, el monómero mayor es de $80 \mathrm{kDa}$, presenta el dominio que produce la proteólisis y es variable según la calpaína, mientras que el monómero de $30 \mathrm{kDa}$ es la subunidad reguladora y está conservada en todas las calpaínas (Huff et al., 2010). La calpaína es la principal enzima del proceso de maduración, donde las variantes más activas de la enzima confieren mayor terneza a la carne (Chung y Davis, 2012).

La calpastatina es una enzima inhibidora de la actividad proteolítica de las calpaínas. Es codificada por el gen CAST, el cual está ubicado en el cromosoma 7 bovino. Este gen expresa cuatro isoformas proteicas diferentes debido a la existencia de cuatro promotores, que se denominan tipo I, II, III y IV. Los transcriptos producen proteínas de 786,697, 
799 y 438 aminoácidos, respectivamente (ENSEMBL, 2017). Se han reportado a la fecha 933 SNPs para bovinos (ENSEMBL, 2017), algunos de los cuales han sido asociados significativamente con la variabilidad en la terneza de la carne bovina (Motter et al., 2009; Chung y Davis, 2012). La calpastatina varía en tamaño y cantidad según la especie animal, lo que influye en el número de moléculas de calpaína inhibidas. El ganado cebuíno se caracteriza por producir mayor cantidad de esta enzima que el taurino, lo que hace que la carne de estas últimas sea más blanda (Barendse et al., 2007).

La selección genética tradicional para mejorar la característica de terneza resulta difícil ya que solo se pueden colectar datos fenotípicos del animal luego del sacrificio (Reardon et al., 2010; Chung y Davis, 2012). La selección de animales basándose en marcadores moleculares asociados a esta característica podría representar una ruta alterna y una herramienta adicional a los esquemas normalmente utilizados en el mejoramiento genético animal (Enriquez-Valencia et al., 2017). Las razas criollas colombianas tienen características importantes, tanto de adaptación como de producción que son potencialmente útiles para contribuir a mejorar los sistemas de producción pecuaria. La raza Hartón del Valle es uno de los ocho grupos raciales de bovinos criollos colombianos y se encuentra en riesgo de extinción (MADR, 2003). En esta raza, se ha estudiado su diversidad genética (Delgado et al., 2011), resistencia genética a enfermedades (Hernández et al., 2014; Bolaños et al., 2017), así como genes relacionados con la calidad de leche (Rosero et al., 2012), aunque en menor profundidad sobre genes asociados a la calidad de la carne (Cuetia et al., 2011; Cuetia, 2012).

En razón a lo antes planteado, el objetivo del presente trabajo fue caracterizar los polimorfismos de nucleótidos simples (SNPs) 316 y 4751 del gen CAPN y 2959 y CAST1 del gen CAST en la raza bovina criolla colombiana Hartón del Valle.

\section{Materiales y Métodos}

\section{Muestras de ADN}

Se trabajó con 126 muestras del banco de ADN del laboratorio de genética animal de la Universidad Nacional de Colombia, sede Palmira, de la raza criolla colombiana Hartón del Valle (HV). Las muestras provenían de 11 fincas de la zona plana del departamento del Valle del Cauca (Colombia).

\section{Amplificación y Genotipificación}

Se amplificaron los SNPs 316 y 4751 del gen Calpaína (CAPN) y 2959 y CAST 1 del gen Calpastatina (CAST). La identificación de los genotipos en los SNPs de los genes CAPN y CAST se realizó mediante PCRRFLP. La PCR se realizó en un volumen final de $25 \mu 1$ que contenían 20 ng de ADN, 1 $\mathrm{U}$ de Taq polimerasa, $1 \mathrm{X}$ de buffer Taq, 2.5 $\mathrm{mM}$ de $\mathrm{MgCl}_{2}, 0.2 \mathrm{mM}$ de cada DNTPs y 2 $\mathrm{mM}$ de cada cebador (Cuadro 1). Los perfiles térmicos constaron de una desnaturalización inicial a $95^{\circ} \mathrm{C}$ durante $5 \mathrm{~min}$, seguido de 35 ciclos de $95^{\circ} \mathrm{C}$ por $60 \mathrm{~s}, 58^{\circ} \mathrm{C}$ por $60 \mathrm{~s}, 72^{\circ} \mathrm{C}$ por $60 \mathrm{~s}$ para CAPN-316; 35 ciclos de $95^{\circ} \mathrm{C}$ por $30 \mathrm{~s}, 64^{\circ} \mathrm{C}$ por $60 \mathrm{~s}, 72^{\circ} \mathrm{C}$ por $60 \mathrm{~s}$ para CAPN-4751; y 30 ciclos de 95 ${ }^{\circ} \mathrm{C}$ por $60 \mathrm{~s}, 60^{\circ} \mathrm{C}$ por $60 \mathrm{~s}, 72{ }^{\circ} \mathrm{C}$ por $60 \mathrm{~s}$ para CAST1 y CAST-2959.

La visualización de los fragmentos amplificados se realizó por electroforesis en geles de agarosa al $1.2 \%$ a $80 \mathrm{~V}$ durante $45 \mathrm{~min}$, teñidos con GelRed ${ }^{\mathrm{TM}}$ (Biotium). Los fragmentos amplificados se utilizaron como sustrato para la digestión con las endonucleasas de restricción que se muestran en el Cuadro 1. Estas reacciones se realizaron en un volumen final de $15 \mu \mathrm{l}$ que contenían en $5 \mu \mathrm{l}$ del producto de PCR, $1 \mathrm{U}$ de cada enzima y $1 \mathrm{X}$ de tampón. La incubación se realizó a $37^{\circ} \mathrm{C}$ durante $3 \mathrm{~h}$, seguido de $80{ }^{\circ} \mathrm{C}$ durante $20 \mathrm{~min}$ para inactivar la enzima. Los productos de digestión se visualizaron en geles de poliacrilamida al 9\% (Acrilamida: Bis-acrilamida 37:1) a $150 \mathrm{~V}$ por 40 min y teñidos con GelRed ${ }^{\mathrm{TM}}$ (Biotium). 


\section{Análisis de los Datos}

Se determinaron las frecuencias genotipicas, alélicas, la heterocigocidad esperada (He) y observada (Ho), el equilibrio de HardyWeinberg (EHW) y el índice de fijación (F). Se realizó un análisis de varianza molecular (AMOVA) considerando cada finca como una subpoblación y se estimó a partir de este el coeficiente de diferenciación genética (FST) y el déficit y/o exceso de heterocigotos dentro (FIS) y entre (FIT) fincas (Bejarano et al., 2012). Todos los análisis se realizaron con los programas GenAIEx v. 6.5 (Peakall y Smouse, 2012) y Arlequin v. 3.5 (Excoffier y Lischer, 2010).

\section{Resultados}

Las frecuencias genotípicas para toda la población de $\mathrm{HV}$ estudiada se presentan en el Cuadro 2. El genotipo más frecuente en el locus CAPN-316 fue el GG, mientras que en los loci CAPN-4751 y CAST-1 fueron los genotipos heterocigotos CT, y en el locus CAST-2959 el genotipo AG. De otro lado, el genotipo de menor frecuencia en el locus CAPN-4751 fue CC, seguido por los genotipos CC en CAPN-316, GG en CAST2959 y CC en CAST-1. En los dos SNPs investigados en el gen CAPN la frecuencia del alelo $\mathrm{C}$ fue más baja (Cuadro 2). Para el locus CAST-1, el alelo T presentó una fre- cuencia alélica de $52.5 \pm 11.4 \%$, mientras que el alelo A fue más frecuente $(0.651 \pm 0.155)$ en el locus CAST-2959.

Teniendo en cuenta los cuatro loci y toda la población de $\mathrm{HV}$, se encontró un valor de heterocigocidad observada de $0.599 \pm 0.248$ $\mathrm{y}$ de $0.410 \pm 0.081$ de heterocigocidad esperada, que tuvo como consecuencia un valor en el índice de fijación de $-0.399 \pm 0.342$, evidenciando un exceso de heterocigotos, aunque sin desviaciones significativas de las proporciones teóricas del equilibrio de HardyWeinberg $(p=0.271)$. En el Cuadro 3 se presentan los valores de $\mathrm{Ho}, \mathrm{He}$, F y la probabilidad de desviación EHW por cada locus.

El índice de fijación mostró valores negativos en todos los marcadores, variando entre $-0.057 \pm 0.095$ en CAPN-316 hasta $0.864 \pm 0.072$ en CAPN-4751. La diversidad genética entendida como la He fue más alta en los loci CAPN-4751 y CAST-1. Solo se encontraron desviaciones significativas de las proporciones teóricas de Hardy-Weinberg en el locus CAPN-4751 ( $\mathrm{p}<0.05)$. El análisis de varianza molecular (Cuadro 4) dividió la variación total en solo dos fuentes: entre fincas y dentro de individuos, siendo esta última la responsable del $97 \%$ de la variación. El valor de $\mathrm{F}_{\mathrm{ST}}$ calculado fue de $0.048(\mathrm{p}<0.05)$. Los valores de $\mathrm{F}_{\text {IS }}$ y $\mathrm{F}_{\text {IT }}$ fueron negativos en ambos casos (-0.411 y -0.343 , respectivamente) sin diferencias significativas.

Cuadro 1. Secuencias de cebadores utilizados, tamaño del amplificado y enzima de restricción utilizada para el genotipado de los cuatro SNPs en estudio

\begin{tabular}{|c|c|c|c|c|}
\hline SNP & Cebador sentido y antisentido (5'a $3^{\prime}$ ) & $\begin{array}{c}\text { Tamaño } \\
(\mathrm{pb})\end{array}$ & $\begin{array}{l}\text { Enzima de } \\
\text { restricción }\end{array}$ & Referencia \\
\hline CAPN-316 & $\begin{array}{l}\text { GGGCCAGATGGTGAACCTGA } \\
\text { TTGCGGAACCTCTGGCTCTT }\end{array}$ & 736 & BTgI & $\begin{array}{l}\text { Page et al. } \\
\quad \text { (2004) }\end{array}$ \\
\hline CAPN-4751 & $\begin{array}{l}\text { AAGGGACAGATGTGGACAGG } \\
\text { GAGGGGTGTTCTCTGAGTGC }\end{array}$ & 550 & BsaJI & $\begin{array}{l}\text { White et al. } \\
\text { (2005) }\end{array}$ \\
\hline CAST-1 & $\begin{array}{l}\text { AGCAGCCACCATCAGAGAAA } \\
\text { TCAGCTGGTTCGGCAGAT }\end{array}$ & 1552 & AluI & $\begin{array}{l}\text { Li et al. } \\
\text { (2013) }\end{array}$ \\
\hline CAST-2959 & $\begin{array}{l}\text { AATATATGCGCTTCCTGGTCTGTCCAG } \\
\text { AATATATTCTCCCCACAGTGCCTGTAA }\end{array}$ & 269 & DdeI & $\begin{array}{l}\text { Barendse } e t \\
\text { al. (2007) }\end{array}$ \\
\hline
\end{tabular}


Cuadro 2. Frecuencias genotípicas y alélicas en cuatro loci en ganado criollo Hartón del Valle

\begin{tabular}{lcc}
\hline Locus & Frecuencias genotípicas & Frecuencias alélicas \\
\hline CAPN-316 & $\mathrm{GG}=0.643 \pm 0.275$ & $\mathrm{G}=0.782 \pm 0.175$ \\
& $\mathrm{CG}=0.278 \pm 0.225$ & ${ }^{1} \mathrm{C}=0.218 \pm 0.175$ \\
CA $=0.079 \pm 0.106$ & \\
& $\mathrm{CC}=0.016 \pm 0.019$ & ${ }^{1} \mathrm{C}=0.484 \pm 0.101$ \\
& $\mathrm{CT}=0.937 \pm 0,196$ & $\mathrm{~T}=0.516 \pm 0.101$ \\
CAST-1 & $\mathrm{TT}=0.048 \pm 0.198$ & \\
& $\mathrm{CC}=0.134 \pm 0.216$ & $\mathrm{C}=0.475 \pm 0.114$ \\
& $\mathrm{CT}=0.683 \pm 0.267$ & \\
CAST-2959 & $\mathrm{TT}=0.175 \pm 0.123$ & ${ }^{1} \mathrm{~A}=0.651 \pm 0.155$ \\
& $\mathrm{AA}=0.389 \pm 0.221$ & $\mathrm{G}=0.349 \pm 0.175$ \\
& $\mathrm{AG}=0.524 \pm 0.153$ & \\
\hline
\end{tabular}

${ }^{1}$ Alelo asociado a terneza de la carne

Cuadro 3. Heterocigocidad observada (Ho), heterocigocidad esperada (He), indice de fijación (F), P-valor de las desviaciones de las proporciones del equilibrio de HardyWeinberg (EHW) por locus en ganado criollo Hartón del Valle

\begin{tabular}{ccc}
\hline Locus & Índice & Promedio \\
\hline CAPN-316 & Ho & $0.335 \pm 0.068$ \\
& He & $0.313 \pm 0.052$ \\
& F & $-0.057 \pm 0.095$ \\
EHWN-4751 & Ho & 0.427 \\
& He & $0.905 \pm 0.005$ \\
& F & $0.478 \pm 0.020$ \\
CAST-1 & EHW & $-0.864 \pm 0.072$ \\
& Ho & 0.049 \\
& He & $0.679 \pm 0.080$ \\
CAST-2959 & FHW & $0.476 \pm 0.006$ \\
& Ho & $-0.412 \pm 0.163$ \\
& He & 0.162 \\
& F & $0.475 \pm 0.046$ \\
& EHW & $0.371 \pm 0.031$ \\
& & $-0.264 \pm 0.033$ \\
\hline
\end{tabular}

${ }^{1} p<0.05$ 
Cuadro 4. Análisis de varianza molecular considerando las 11 fincas de donde provenían las muestras de ADN del ganado criollo Hartón del Valle

\begin{tabular}{lccc}
\hline Fuente de variación & G.L. & CM & Variación (\%) \\
\hline Entre fincas & 10 & 1.440 & 3 \\
Entre individuos & 115 & 0.497 & 0 \\
Dentro de individuos & 126 & 1.190 & 97 \\
Total & 251 & & 100 \\
\hline
\end{tabular}

\section{Discusión}

En este mismo grupo racial, Cuetia (2012) reporta una frecuencia de $4 \%$ para el genotipo CC del locus CAPN-316 y frecuencias menores al $5 \%$ en las razas criollas colombianas Romosinuano, San Martinero, Caqueteño, Chino Santandereano y Casanareño; mientras que en las razas criollas de Bolivia se encontró 5\% de frecuencia para este genotipo en las razas Chaqueño y Yacumeño y $12 \%$ para la raza Saavedreño (Pereira et al., 2015). Por otro lado, no se encontró el genotipo CC de este locus en las razas Hereford, Limousin, Simmental (Li et al., 2013) y Nellore (Pinto et al., 2010), pero se encontró en las razas Angus (11\%) y Charolais (4\%) (Pinto et al., 2010). En general, el genotipo CC presenta frecuencia genotípica similar en todos los reportes, al igual que el genotipo GG, el cual es el más frecuente en todas las razas mencionadas.

En el locus CAPN-4751, Cuetia (2012) reporta una frecuencia de $25 \%$ para el genotipo $\mathrm{CC}$ en animales $\mathrm{HV}$, diferente al encontrado en este estudio $(0.016 \pm 0.019)$, posiblemente debido a las diferencias en tamaño de muestra $(n=30)$ usado por este autor, que a su vez presenta altas frecuencias en este genotipo en todas las razas criollas colombianas analizadas; no obstante, en otro estudio, también en HV, presenta similar frecuencia del genotipo CC (Cuetia et al., 2011).
Frecuencias menores de 5\% se reportan en animales cruzados Angus x Nellore y en Rubia Gallega x Nellore (Curi et al., 2009). De otro lado, este autor no encontró el genotipo $\mathrm{CC}$ en animales Nellore, aunque Pinto et al. (2010) reportan una frecuencia de $3.2 \%$ en esta raza. Respecto al genotipo TT, los autores antes mencionados lo refieren como el más frecuente, lo cual difiere de este reporte y lo presentado por Pereira et al. (2015) en el criollo de Bolivia; en tanto que Ribeca et al. (2012) en ganado de raza Piemontese y Cuetia (2012) en ocho razas criollas colombianas presentan al genotipo CT como el más frecuente $(50 \%)$.

El genotipo heterocigoto CT del locus CAST-1, al igual que en el presente reporte, fue el más frecuente en las razas Charolais, Hereford y Limousin (Li et al., 2013), al igual que en ocho razas criollas colombianas (Cuetia, 2012); mientras que el homocigoto TT lo fue en Angus (Li et al., 2013) y en el criollo colombiano Costeño con Cuernos (Cuetia, 2012), y el genotipo CC en Simmental (Li et al., 2013).

En animales cruzados Angus x Nellore y Rubia Gallega x Nellore no se encontró el genotipo GG del locus CAST-2959 y esta frecuencia fue más alta en animales Nellore (22\%) (Curi et al., 2009) que en las razas Piemontese (10\%) (Ribeca et al., 2012), criollo colombiano (12\%) (Cuetia et al., 2011), 
Hartón del Valle (10\%) (Cuetia, 2012) y en la presente investigación ( $8.7 \%$ ). De otro lado, el genotipo AA presentó en promedio mayor al $25 \%$ en todos los reportes antes mencionados.

El SNP CAPN-316 es una transversión citosina por guanina $(\mathrm{C} / \mathrm{G})$, donde el alelo $\mathrm{C}$ codifica para el aminoácido alanina y el alelo G codifica para glicina, mientras que el locus CAPN-4751 es una transición citosina por timina $(\mathrm{C} / \mathrm{T})$. En ambos loci, el alelo $\mathrm{C}$ ha sido asociado con terneza de la carne (White et al., 2005; Allais et al., 2011; Sava ${ }^{\circ} c ̧ i \mathrm{y}$ Atasoy, 2016). Aunque la frecuencia de estos alelos en HV no fue la más alta, presenta frecuencias mayores al 21\% (Cuadro 2), lo cual falicitaría su selección en programas de mejoramiento genético. Por su parte, Li et al. (2013) muestran frecuencias para el alelo $\mathrm{C}$ de $36 \%$ para el locus CAPN-316 en Angus, $14 \%$ en Charolais, $3 \%$ en Hereford, $16 \%$ en Limousin y $17 \%$ en Simmental, frecuencia solo superada por Angus en comparación con el HV. Castro et al. (2016) para este mismo locus reportan frecuencias de 10\% para el alelo $\mathrm{C}$ en animales Brahman y Pinto et al. (2010) del 8\% en Nellore. Frecuencia similar a la encontrada en el HV la reportan Pereira et al. (2015) en tres razas de ganado criollo boliviano y Cuetia (2012) en ocho razas criollas colombianas. De otro lado, el alelo $\mathrm{C}$ en el locus CAPN-4751 en el HV mostró mayor frecuencia que lo reportado en animales Nellore y cruzados Angus x Nellore y Rubia Gallega x Nellore (Curi et al., 2009), en Nellore (Pinto et al., 2010) y en tres razas criollas colombianas (Cuetia, 2012). Sin embargo, las frecuecias de este alelo son superiores al $60 \%$ en los grupos genéticos trabajados por Castro et al. (2016) y Pereira et al. (2015).

En el gen CAST el marcador CAST-1 es una transición de timina por citosina (T/C)y en el marcador CAST-2959 la mutación es una transición de guanina por adenina (G/A) en la región 3' no traducida del gen. Los alelos asociados con terneza de la carne se señalan en el Cuadro 2 (Juszczuk-Kubiak et al., 2008;
Bosques et al., 2015). Es importante resaltar que las frecuencias de los alelos favorables para estos genes fueron altas en el ganado $\mathrm{HV}(\mathrm{T}=52.5 \%$ en CAST -1 y A $=65.1 \%$ en CAST-2959). El locus CAST-1 en las razas Angus, Charolais, Hereford, Limousin y Simmental tuvieron frecuencias para el alelo $\mathrm{T}$ de 79, 57, 59, 57, 24 y 58\%, respectivamente (Li et al., 2013). Así mismo, Chung y Davis (2012) reportan una frecuencia de $42.9 \%$ en ganado Hanwoo para este alelo, mientras que Castro et al. (2016) obtienen frecuencias menores al $35 \%$ en animales Brahman y cruces de Bos indicus x $B$. taurus. Ribeca et al. (2012) en ganado Piemontese, Castro et al. (2016) en ganado Brahman, Cuetia et al. (2011) en ganado criollo colombiano y Cuetia (2012) en ganado HV presentan una frecuencia similar al presente estudio (65.1\%) para el alelo A para el locus CAST-2959, en tanto que Curi et al. (2009) reporta una mayor frecuencia en animales cruzados Angus x Nellore (89\%) y Rubia Gallega $x$ Nellore (82\%) y menor en animales Nellore (55\%).

Pereira et al. (2015) muestra valores de He para los loci CAPN-316/CAPN-4751 en las razas criollas Bolivianas Chaqueño de $0.36 / 0.38$, Yacumeño de $0.35 / 0.37 \mathrm{y}$ Saavedreño de $0.45 / 0.48$, que son similares al presente trabajo, pero muestra déficit de heterocigotos en ambos loci en la raza Chaqueño y exceso en las otras dos. Castro (2013) reporta un valor bajo de He en ganado Brahman (0.087) para el locus CAPN316 y de 0.21 para el locus CAPN-4751, sin desviaciones en EHW para ambos loci. Cuetia (2012) en 30 muestras de HV reporta un exceso no significativo de heterocigotos en los loci CAPN-4751, CAST-1 y CAST2959, y un déficit en el locus CAPN-316 $(-0.166, p>0.05)$. Así mismo, Cuetia (2012) reporta que no hay desviaciones de EHW en ningún loci, lo cual no concuerda con lo aquí presentado. Estas variaciones en los valores de Ho, He y EHW entre esta investigación y las otras razas criollas podrían deberse al tamaño de muestra utilizado, diferencias en los procesos de formación de las razas y a pro- 
cesos de selección a favor de alguno de los loci estudiados.

Cuetia (2012) simuló diferentes estructuras genéticas para todas las razas criollas colombianas, encontrando siempre que la mayor variación se encontraba dentro de los individuos, lo que concuerda con esta investigación y explica a su vez el bajo valor del coeficiente de diferenciación genética $\left(\mathrm{F}_{\mathrm{ST}}\right)$ encontrado. El $\mathrm{F}_{\mathrm{ST}}$ cuantifica la consanguinidad de las subpoblaciones con relación a la población total de la cual forma parte, y se interpreta como la proporción de la variación total atribuible a la diferenciación entre las poblaciones (Bejarano et al., 2012). El valor encontrado indica poca diferenciación genética entre las fincas, lo que sugiere que es una sola gran población donde hay flujo de genes, y que no se ha realizado una selección del conjunto de genes que confieren atributos a la calidad de la carne.

Los estadísticos $\mathrm{F}_{\text {IS }}$ y $\mathrm{F}_{\text {IT }}$ miden de la desviación de las frecuencias genotípicas, en términos de exceso o deficiencia de heterocigotos, dentro de una subpoblación y entre un grupo de poblaciones, respectivamente. Convencionalmente, se definen como la probabilidad de que dos alelos en un individuo sean idénticos por descendencia respecto a la subpoblación. El valor de este índice varía entre -1 y 1 . Cuando tiende a 0 significa que la población está cerca del equilibrio de EHW. Los valores negativos indican exceso de heterocigotos, mientras que un valor positivo indica una deficiencia de estos (Bejarano et al., 2012). En el presente trabajo ambos índices fueron negativos y sin diferencia significativa de cero, lo cual concuerda con los resultados de Cuetia (2012) y Castro (2013) en ganado Brahman.

\section{Conclusiones}

- Las frecuencias genotípicas y alélicas para el ganado colombiano criollo Hartón del Valle (HV) fueron similares a las reportadas en varias razas criollas y especializadas en producción de carne con altas frecuencias de los alelos asociados a terneza.

- Existe un leve exceso de genotipos heterocigotos, pero la variación a nivel de individuo fue muy alta, indicando que en el HV no han llevado a cabo procesos de selección a favor de alguno de los marcadores analizados.

- El alto flujo genético entre los hatos de $\mathrm{HV}$ fue evidenciado en el valor de $\mathrm{F}_{\mathrm{ST}}$. Todo lo anterior puede ser indicativo que el HV no solo presenta características favorables de adaptación a las condiciones ambientales, sino que también presenta una predisposición genética para la producción de carne tierna.

\section{Literatura Citada}

1. Allais S, Journaux L, Lezeviel H, Payet-Duprat $N$, Raynaud $P$, Hocquette J, et al. 2011. Effects of polymorphisms in the calpastatin and icalpain genes on meat tenderness in 3 French beef breeds. J Anim Sci 89: 111. doi:10.2527/jas.2010-3063

2. Ba HV, Reddy BV, Hwang I. 2015. Role of calpastatin in the regulation of mRNA expression of calpain caspase and heat shock protein systems in bovine muscle satellite cells. In Vitro Cell Dev Biol Anim 51: 447-454. doi: 10.1007/ s11626-014-9849-8

3. Barendse W, Harrison B, Hawken R, Ferguson D, Thompson J, Thomas M, Bunch RJ. 2007. Epistasis between calpain 1 and its inhibitor calpastatin within breeds of cattle. Genetics 176: 2601-2610. doi: 10.1534/genetics. 107.074328

4. Bejarano D, Pedraza A, Rocha J, Martínez R. 2012. Genetic variability in commercial subpopulations of creole colombian breed Romosinuano. Rev Corpoica Cienc Tecnol Agropec 13(1): 97-107. 
5. Bolaños I, Hernández D, Álvarez LA. 2017. Asociación de los alelos del gen BoLA-DRB3 con la infección natural de Babesia spp en el ganado criollo Hartón del Valle. Arch Zootec 66: 113-120. doi: org/10.21071/az.v66i253.2133

6. Bosques J, Pagan-Morales M, Casas A, Rivera A, Cianzio D. 2015. Efecto de los polimorfismos en m-calpaína y calpastatina en características de importancia económica en ganado para carne en Puerto Rico. JAgrie UnivPR 99: 87-104.

7. Castro S, Ríos M, Ortiz Y, Manrique C, Jimenez A, Ariza F. 2016. Association of single nucleotide polymorphisms in CAPN1 CAST and -MB genes with meat color of Brahman and crossbreed cattle. Meat Sci 117: 44-49. doi: org/ 10.1016/j.meatsci.2016.-02.021

8. Castro S. 2013. Evaluacion de polimorfismos de nucleotido simple asociados a cambios en el color de la carne en ganados Bos indicus y cruces con Bos taurus. Tesis de Maestría. Colombia: Univ. Nacional de Colombia. $115 \mathrm{p}$.

9. Chriki S, Renand G, Picard B, Micol D, Journaux L, Hocquette J. 2013. Meta-analysis of the relationships between beef tenderness and muscle characteristics. Livest Sci 155: 424-434. doi: 10.1016/j.livsci.2013.04.009

10. Chung H, Davis M. 2012. Effects of genetic variants for the calpastatin gene on calpastatin activity and meat tenderness in Hanwoo (Korean cattle). Meat Sci 9: 711-714. doi: 10.1016/ j.meatsci.2011.10.017

11. Cuetia J, Posso A, Hernadez D, Ariza M, Muñoz J, Alvarez. L. 2011. Polimorfismos de los genes calpaina y calpastatina en diez razas bovinas criollas mediante siete marcadores de polimorfismo de nucleótidos simple (SNPs). AICA 1: 191-194.

12. Cuetia J. 2012. Polimorfismo de los genes calpaína, calpastatina y leptina en diez razas bovinas criollas mediante siete marcadores de polimorfismos de nucleótido simple (SNPs). Tesis de
Maestría. Colombia: Univ. Nacional de Colombia. $81 \mathrm{p}$.

13. Curi R, Chardulo L, Mason M, Arrigoni M, Silveira A, de Oliveira $H$. 2009. Effect of single nucleotide polymorphisms of CAPN1 and CAST genes on meat traits in Nellore beef cattle (Bos indicus) and in their crosses with Bos taurus. Anim Genet 40: 456-462. doi: 10.1111/j.1365-2052.2009.01859.x

14. Delgado JV, Martínez AM, Acosta A, Álvarez LA, Armstrong E, Camacho E, Cañón J, et al. 2011. Genetic characterization of Latin-American creole cattle using microsatellite markers. Anim Genet 43(1): 2-10. doi:10.1111/ j.1365-2052.2011.02207.x

15. Enriquez-Valencia C, Pereira L, Malheiros J, de Vasconcelos Silva JA, Albuquerque LG, de Oliveira HN, et al. 2017. Effect of the g.98535683ANG SNP in the CAST gene on meat traits of Nellore beef cattle (Bos indicus) and their crosses with Bos taurus. Meat Sci 123: 64-66. doi: 10.1016/j.meatsci.2016.09.003

16. ENSEMBL 2017. European Bioinformatics Institute. [Internet]. Disponible en: https://www.ensembl.org/index.html

17. Excoffier L, Lischer H. 2010. Arlequin suite ver 3.5: a new series of programs to perform population genetics analyses under Linux and Windows. Mol Ecol Resour 10: 564-567. doi: 10.1111/j.17550998.2010.02847.x

18. Hernández D, Muñoz JE, Álvarez LA. 2014. Asociación del locus BoLADRB3.2 con el virus de la leucosis bovina en el ganado criollo colombiano. Rev Colomb Cienc Anim 6: 319-326.

19. Hill R, Canal A, Bondioli K, Morell R, Garcia M. 2016. Molecular markers located on the DGAT1 CAST and LEPR genes and their associations with milk production and fertility traits in Holstein cattle. Genet Mol Res 15: 1-8. doi: 10.4238/gmr.15017794

20. Huff E, Zhang W, Lonergan S. 2010. Biochemistry of postmortem muscle lessons on mechanisms of meat 
tenderization. Meat Sci 86: 184-195. doi: 10.1016/j.meatsci.2010.05.004

21. Juszczuk-Kubiak E, Wyszynska-Koko J, Wicinska K, Rosochacki S. 2008. A novel polymorphisms in intron 12 of the bovine calpastatin gene. Mol Biol Rep 35: 29-35. doi: 10.1007/s11033-006-9048-0

22. Li X, Ekerljung M, Lundström K, Lundén A. 2013. Association of polymorphisms at DGAT1, leptin, SCD1, CAPN1 and CAST genes with color, marbling and water holding capacity in meat from beef cattle populations in Sweden. Meat Sci 94: 153-158. doi: 10.1016/j.meatsci.2013.01.010

23. [MADR] Ministerio de Agricultura y Desarrollo Rural. 2003. Situación actual de los recursos Zoogenéticos en Colombia. [Internet]. Disponible en: http:// bibliotecadigital.agronet.gov.co/handle/ 11348/3952

24. Motter M, Corva P, Krause M, Perez Cenci M, Soria L. 2009. Rol de la calpastatina en la variabilidad de la terneza de la carne bovina. BAG J Basic Appl Genet 20: 15-24.

25. Page BT, Casas E, Quaas RL, Thallman RM, Wheeler TL, Shackelford SD, Koohmaraie M, et al. 2004. Association of markers in the bovine CAPN1 gene with meat tenderness in large crossbred populations that simple influential industry sires. J Anim Sci 82: 3474-3481. doi: 10.2527/ 2004.82123474x

26. Peakall R, Smouse P. 2012. GENALEX 6.5: genetic analysis in Excel. Population genetic software for teaching and research - an update. Bioinformatics 28: 2537-2539. doi: 10.1093/bioinformatics/bts460

27. Pereira J, Falomir-Lockhart A, Loza A, Villegas E, Rojas P, Carino M, Ripoli M, et al. 2015. Comparación de frecuencias alélicas y genotípicas de los polimorfismos CAPN1-316 y CAPN14751 del gen de la calpaína en tres poblaciones de ganado criollo boliviano. AICA 6: 156-164.

28. Pinto L, Ferraz J, Meirelles F, Eler J, Rezende F, Carvalho M, et al. 2010. Association of SNPs on CAPN1 and CAST genes with tenderness in Nellore cattle. Genet Mol Res 9: 1431-1442. doi: 10.4238/vol9-3gmr881

29. Reardon W, Mullen A, Sweeney T, Hamill R. 2010. Association of polymorphisms in candidate genes with colour, water-holding capacity, and composition traits in bovine $\mathrm{M}$ longissimus and $\mathrm{M}$ semimembranosus. Meat Sci 86: 270-275. doi: 10.1016/j.meatsci.2010.04.013

30. Ribeca C, Bonfatti V, Cecchinato A, Albera A, Maretto F, Gallo L, Carnier P. 2012. Association of polymorphisms in calpain $1(\mathrm{mu} / \mathrm{I})$ large subunit calpastatin and cathepsin $\mathrm{D}$ genes with meat quality traits in double-muscled Piemontese cattle. Anim Genet 44: $193-$ 196. doi: 10.1111/j.1365-2052.2012.02370.x

31. Rosero J, Álvarez LA Muñoz JE, Durán C, Rodas A. 2012. Allelic frequency of the Kappa-Casein gene in Colombian and creole cattle breeds. Rev Colomb Cienc Pecu 25: 173-182.

32. Sava'çi M, Atasoy F. 2016. The investigation of calpastatin and thyroglobulin gene polymorphisms in some native cattle breeds. Ankara Üniv Vet Fak Derg 63: 53-59. doi: 10.1501/ Vetfak 0000002709

33. White S, Casas E, Wheeler T, Shackelford S, Koohmaraie M, Riley D, Chase $C$, et al. 2005. A new single nucleotide polymorphism in CAPN1 extends the current tenderness marker test to include cattle of Bos indicus, Bos taurus, and crossbred descent. J Anim Sci 83: 2001 2008. doi: $10.2527 / 2005.8392001 x$ 